\title{
Vaccine development: baring the 'dirty little secret'
}

\author{
David L Woodland \& Marcia A Blackman
}

Inflammatory signals strongly influence the generation of T-cell memory after infection or vaccination. Experimental manipulation of these signals shortens the interval of time between administration of a vaccine and a booster (pages 748-756).

Vaccines designed to elicit cellular immunity typically require a secondary boost to generate protective numbers of memory $\mathrm{T}$ cells. For the boost to be effective, it has to be delivered weeks or months after the initial vaccination, a problem when rapid protective responses are required. In this issue, Badovinac et al. show how inflammatory signals influence Tcell priming (the activation, differentiation and proliferation of $\mathrm{T}$ cells) and the rate at which memory $\mathrm{T}$ cells develop ${ }^{1}$. Appropriate manipulation of these inflammatory signals during vaccination accelerates the generation of stable T-cell memory and facilitates much earlier boosting of the response.

An important characteristic of the immune system is its ability to remember past encounters with a pathogen and use that information to mount improved responses to a secondary challenge with the same pathogen ${ }^{2}$. This property of immune memory is harnessed by vaccines that promote the development of memory B and T cells through controlled exposure to pathogen-derived antigens.

One particularly effective vaccination strategy is to prime and subsequently boost the T-cell response using antigens presented in the context of different delivery vectors ${ }^{3}$. This increases the number of memory T cells generated and consequently results in increased protective efficacy. In addition, effective $\mathrm{T}$ cell vaccination requires the induction of an inflammatory response, typically initiated by adjuvants, termed "the immunologist's dirty little secret" by Charles Janeway, Jr. ${ }^{4}$.

Great progress has been made in characterizing the inflammatory signals required to generate potent T-cell memory. These signals act both at the level of T-cell priming, by activating antigen-presenting dendritic cells that drive the priming, and at the level of T-cell proliferation and apoptosis, by regulating the numbers of memory $\mathrm{T}$ cells ultimately generated. During this second phase, both the expansion of the $\mathrm{CD}^{+}{ }^{+} \mathrm{T}-$ cell response and its subsequent contraction

The authors are at theTrudeau Institute, Saranac Lake, New York 12983, USA.

e-mail: dwoodland@trudeauinstitute.org

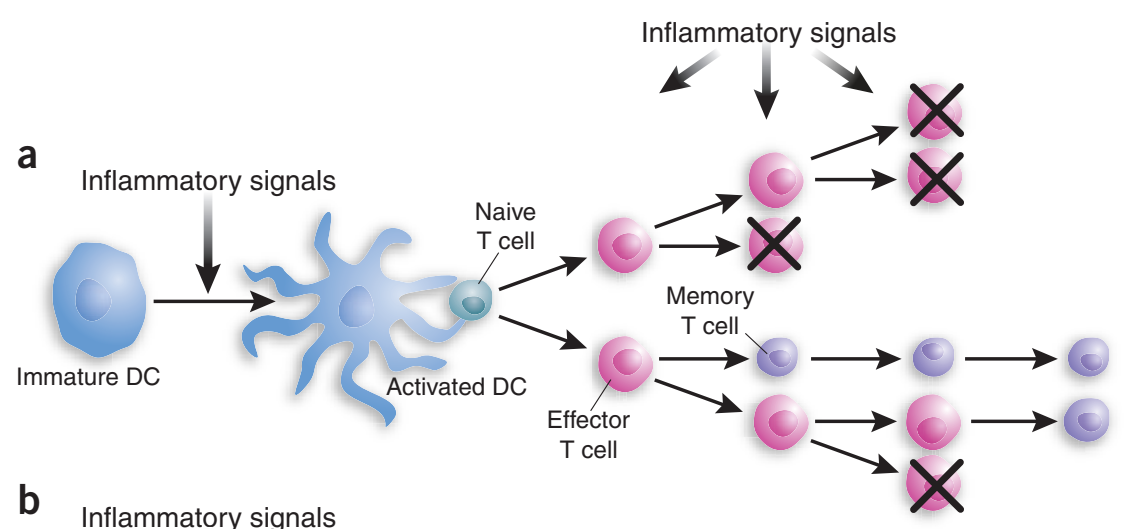

Figure 1 Inflammatory signals affect the development of memory T cells. Early inflammatory signals are required to mediate the activation of immature dendritic cells (DC) and promote the presentation of antigen to naive T cells. Naive T cells that have been primed by DCs undergo a program of activation and proliferation which culminates in the development of memory T cells. (a) Inflammatory signals (including IFN- $\gamma$ ) present during this latter phase of the response act to both enhance the proliferation of effector T cells and also promote the contraction of the response through apoptosis (b). The absence of inflammatory signals during the T-cell expansion phase reduces the overall amplification and contraction of the response and promotes the early development of memory $T$ cells. The rapid development of stable memory $T$ cells makes it possible to effectively boost the memory T-cell pools much sooner after the initial priming, as shown by Badovinac et al ${ }^{1}$.

into a memory T-cell pool are driven, in part, by interferon (IFN)- $\gamma^{5,6}$.

Memory $\mathrm{T}$ cells appear very early during the primary response ${ }^{7}$. But a relatively long waiting period between the priming and boosting phases normally is required to elicit protective immunity, presumably to allow the resolution of inflammation and the generation of stable memory T cells. Badovinac et al. could drastically shorten this waiting period by manipulating the inflammatory conditions during the initial vaccination.

The authors focused on developing immunity against the bacterium Listeria monocytogenes. They infected one group of mice with the bacterium and vaccinated another group with in vitro-generated dendritic cells coated with a dominant $L$. monocytogenes peptide. Both approaches elicited $\mathrm{CD} 8^{+}$memory $\mathrm{T}$ cells that could be boosted by a secondary infection with the bacterium. But effective boosting of the response could be achieved as early as 4 days after dendritic-cell vaccination, compared to more than 11 days after L. monocytogenes infection.

This dramatically shortened interval was not due to differences in the rate of clearance of the boosting antigen or to the presence or absence of antibodies to the bacterium. Rather, it appeared to result from the early development of stable memory $\mathrm{CD}^{+} \mathrm{T}$ cells after dendriticcell vaccination. The authors attributed the early memory T-cell development in this case to the expansion of dendritic cell-primed $\mathrm{T}$ cells in the absence of concurrent inflammation. Consistent with this, administration of an inflammatory stimulus (CpG oligodeoxynucleotides) after dendritic-cell vaccination delayed the generation of stable memory $\mathrm{T}$ cells and lengthened 
the interval until boosting could be achieved. This CpG oligonucleotide-mediated delay in stable memory T-cell generation was reversed when the T cells lacked IFN- $\gamma$ receptors, suggesting a key role for IFN- $\gamma$ in the generation of memory $\mathrm{CD}^{+} \mathrm{T}$ cells.

The direct action of IFN- $\gamma$ on $\mathrm{CD}^{+} \mathrm{T}$ cells is consistent with recently reported changes in the expression of the IFN- $\gamma$ receptor and in IFN- $\gamma$ ๖ responsiveness by $\mathrm{T}$ cells, which wane during the expansion and increase during contraction phases of the effector T-cell response ${ }^{8}$. The current observations distinguish the effects of inflammatory signals on dendritic cells and $\mathrm{T}$ cells. Whereas early inflammatory signals activate dendritic cells and initiate T-cell priming, inflammation during the T-cell expansion phase of the response delays the generation of stable T-cell memory and lengthens the time before these cells can be effectively boosted (Fig. 1).

The differential targeting of dendritic cells and $T$ cells by inflammatory signals is crucial to understand from a vaccine standpoint.

Current adjuvants induce generalized inflammation that may not elicit optimal memory T-cell generation and may also have numerous side effects, such as local swelling and fever.

Badovinac et al. begin to define the specific actions of inflammation during vaccination, opening the door to vaccine strategies capable of targeting inflammatory responses that optimize T-cell memory development without generalized inflammation ${ }^{9}$. Because vaccination strategies based on in vitro-cultured dendritic cells may have limited utility in the clinic, an alternative strategy may be to specifically target inflammatory signals to the dendritic cell in vivo. One promising approach, promoted by Steinman and colleagues, is to limit antigen delivery and inflammatory signals to the dendritic cell in vivo using antibodies targeted to these cells ${ }^{10}$. In this case, the antigen is delivered through an antibody to the DEC-205 receptor on dendritic cells and inflammation is elicited by an agonistic CD40specific antibody.

The coupled targeting of antigen delivery and limited inflammatory signals to dendritic cells is also a feature of DNA vaccines and may explain why they are able to prime memory $\mathrm{T}$ cells without inducing the expansion of large effector populations.

Many questions remain about inflammation during vaccination. Although IFN- $\gamma$ is clearly a key mediator, what other inflammatory signals operate at each stage of the response? How does inflammation affect the development of memory $\mathrm{CD}^{+} \mathrm{T}$ cells that mediate protection at peripheral or mucosal sites? What is the role of inflammation on $\mathrm{CD} 4^{+} \mathrm{T}$-cell responses and the delivery of help required for the establishment of long-lasting $\mathrm{CD}^{+} \mathrm{T}$-cell memory ${ }^{11}$ ? Finally, inflammation could favor the generation of regulatory $\mathrm{CD} 4^{+} \mathrm{T}$ cells, which may dampen the efficacy of memory $\mathrm{CD}^{+} \mathrm{T}$ cells ${ }^{12}$. Clearly, much remains to be done, but this work lays a strong foundation for future studies.

1. Badovinac, V.P., Messingham, K.A.N., Jabbari, A., Haring, J.S. \& Harty, J.T. Nat. Med. 11, 748-756 (2005).

2. Seder, R.A. \& Ahmed, R. Nat. Immunol. 4, 835-842 (2003).

3. Woodland, D.L. Trends Immunol. 25, 98-104 (2004).

4. Janeway, C.A., Jr. Cold Spring Harb. Symp. Quant. Biol. 54 Pt 1, 1-13 (1989).

5. Badovinac, V.P., Tvinnereim, A.R. \& Harty, J.T. Science 290, 1354-1358 (2000).

6. Whitmire, J.K., Tan, J.T. \& Whitton, J.L. J. Exp. Med 201, 1053-1059 (2005).

7. Wong, P., Lara-Tejero, M., Ploss, A., Leiner, I. \& Pamer, E.G. J. Immunol. 172, 7239-7245 (2004).

8. Haring, J.S., Corbin, G.A. \& Harty, J.T. J. Immunol. 174, 6791-6802 (2005).

9. Dudani, R. et al. Infect. Immun. 70, 1957-1964 (2002).

10. Bonifaz, L.C. et al. J. Exp. Med. 199, 815-824 (2004).

11. Bevan, M.J. Nat. Rev. Immunol. 4, 595-602 (2004).

12. Yamazaki, S. et al. J. Exp. Med. 198, 235-247 (2003).

\title{
Immune intervention with anti-CD3 in diabetes
}

\author{
David M Harlan \& Matthias von Herrath
}

Autoimmune processes that destroy insulin-producing cells in the pancreas cause type 1 diabetes. To prevent the disease, autoreactive immune cells need to be suppressed or eliminated without deleterious side effects. Results from a phase 2 clinical trial take steps in this direction.

Type 1 diabetes mellitus is caused by genetic and environmental factors that conspire to promote autoimmune-mediated loss of insulin-producing beta cells in the pancreas. This disease commonly strikes children or young adults, and is costly, chronic and, for too many, disabling. Beta-cell loss can be prevented by general immunosuppression ${ }^{1}$, but

David M. Harlan is in the NIDDK Islet and Autoimmunity Branch, National Institutes of Health, Bethesda, Maryland 20812, USA. Matthias von Herrath is at the La Jolla Institute for Allergy and Immunology, Immune Regulation Lab, San Diego, California 92121, USA.

e-mail: davidmh@mail.nih.gov or matthias@liai.org past approaches had unacceptable side effects. Because reasonable life quality and expectancy can be afforded with modern insulin therapy ${ }^{2}$, the ethical bar for any intervention is set high.

Previous studies by Herold and colleagues have shown promise using an antibody directed against the CD3 molecule expressed on most $\mathrm{T}_{\text {cells }}{ }^{3}$. Keymeulen et al. have now extended these results in a controlled trial of 80 individuals with recent-onset type 1 diabetes. Infusion of a different CD3-specific antibody maintained insulin production over 18 months, as reported in a recent issue of the New England Journal of Medicine ${ }^{4}$.

Experimental evidence strongly suggests that $\mathrm{T}$ cell-mediated beta-cell killing is relentless once underway. Over 20 years ago, Sutherland and colleagues studied whether living identical twin women 'discordant' for type 1 diabetes could serve as distal pancreas donors for their diabetic sisters, in hopes that the recipient could avoid immunosuppression therapy. Unfortunately, in all three pairs reported, the recipient's autoreactive $\mathrm{T}$ cells specifically destroyed the graft islets within 12 weeks ${ }^{5}$.

Hope arose in 1994 with reports that antibody specific for mouse CD3 could permanently restore normal blood sugar levels in most nonobese diabetic (NOD) mice, when administered shortly after disease onset ${ }^{6}$. Much subsequent NOD mouse studies suggested that the CD3-specific antibody worked, at least in part, by promoting the function of protective regulatory $\mathrm{T}$ cells - thereby shield- 